TRANSACTIONS OF THE

AMERICAN MATHEMATICAL SOCIETY

Volume 352, Number 8 , Pages 3777-3798

S 0002-9947(00)02561-7

Article electronically published on April 13, 2000

\title{
MEAN CONVERGENCE OF ORTHOGONAL FOURIER SERIES OF MODIFIED FUNCTIONS
}

\author{
MARTIN G. GRIGORIAN, KAZAROS S. KAZARIAN, AND FERNANDO SORIA
}

\begin{abstract}
We construct orthonormal systems (ONS) which are uniformly bounded, complete, and made up of continuous functions such that some continuous and even some arbitrarily smooth functions cannot be modified so that the Fourier series of the new function converges in the $L^{p}$-metric for any $p>2$. We prove also that if $\Phi$ is a uniformly bounded ONS which is complete in all the spaces $L_{[0,1]}^{p}, 1 \leq p<\infty$, then there exists a rearrangement $\sigma$ of the natural numbers $\mathbf{N}$ such that the system $\Phi_{\sigma}=\left\{\phi_{\sigma(n)}\right\}_{n=1}^{\infty}$ has the strong $L^{p}$-property for all $p>2$; that is, for every $2 \leq p<\infty$ and for every $f \in L_{[0,1]}^{p}$ and $\epsilon>0$ there exists a function $f_{\epsilon} \in L_{[0,1]}^{p}$ which coincides with $f$ except on a set of measure less than $\epsilon$ and whose Fourier series with respect to the system $\Phi_{\sigma}$ converges in $L_{[0,1]}^{p}$.
\end{abstract}

\section{INTRODUCTION}

In his famous dissertation $[\mathrm{L}]$, N. Luzin proved that for every measurable and almost everywhere finite function $f$ on the circle $\partial B=\{|z|=1\}$ there is a harmonic function $U(r, t)$ in the $\operatorname{disc} B=\{|z|<1\}$ such that

$$
\lim _{r \rightarrow 1-} U(r, t)=f\left(e^{i t}\right) \quad \text { a.e. }
$$

The proof of the above assertion is based on Luzin's well known C-property: "For every measurable, almost everywhere finite function $f$ on $[0,1]$ and every $\epsilon>0$ there is a perfect set $E_{\epsilon} \subset[0,1]$ on which $f$ is continuous and $\left|E_{\epsilon}\right|>1-\epsilon . "$

This theorem establishes a connection between the concepts of measurability and continuity of functions. It shows that measurable functions are continuous outside of sets with arbitrarily small measure. Luzin's C-property was not only the first theorem in the series of "correction" assertions, but it also showed for the first time how a modification result could be used for proving a so-called representation theorem. Of course the problem of the representation of functions was studied before Luzin's work. It goes back to D. Bernoulli, L. Euler and many others. The modification of functions by a change of the values of the given function on a suitable set of arbitrarily small measure became an independent area of investigation after Men'shov's proof [M2] of the so-called C-strong property (see also [B], pp. 500-510):

Received by the editors November 26, 1997.

2000 Mathematics Subject Classification. Primary 42C15, 42C20.

Key words and phrases. Complete orthonormal system, C-strong property, modification of functions, rearrangements of systems, divergence in metric, universal series.

The research described in this publication was made possible in part by Grant PB97/0030 from DGES. The first two authors were also supported by Grant MVR000 from the I.S.F. 
"For every measurable almost everywhere finite function $f$ on $[0,2 \pi]$ and every $\epsilon>0$ there is a continuous function $f_{\epsilon}$ such that $\left|\left\{x: f_{\epsilon}(x) \neq f(x)\right\}\right|<\epsilon$ and the Fourier series of the function $f_{\epsilon}$ converges uniformly."

This result was used later on by Men'shov [M3 in order to give an answer to a problem posed by Luzin. Namely, he proved that every measurable almost everywhere finite function can be represented by a series of the trigonometric system converging to $f$ almost everywhere.

What Luzin had in mind by posing that problem was not only how to clarify whether it was possible to strengthen his own result. Mainly, he was trying to define a new, more general integral. He was convinced, somehow, that if a function is represented by a.e. convergent trigonometric series, then the coefficients of the series must be unique. This hope was ruined by Men'shov's construction of a trigonometric series with nonzero coefficients which converges to zero a.e. (see M1]). Probably this is a good occasion to formulate a problem which Luzin called Fourier's Problem ([L], pp. 49-51), because it was one of the strong motivations for him to pose problems which would stimulate investigations on representations of functions by trigonometric and other orthogonal series, and because up to now not much has been done for its solution. A literal translation of his statement follows:

Fourier's Problem. Given an arbitrary measurable function by its values, determine the coefficients of a trigonometric series which represents it.

After Men'shov's proof of the C-strong property, many "correction" type theorems were proved for different systems. We are not going to give a complete survey of all the research done in this area. The interested reader can find references in $[\mathrm{GW}],[\mathrm{U}]$ and $[\mathrm{Gr}]$. The results of our article can be divided into two parts - results of "positive" and "negative" type. In the first part of our article (sections 1.2 and 1.3) we will give results of "negative" type and in the second part (section 2.2) "positive" type. This order is chosen mainly because results of "negative" type were proved first by two of the coauthors (see [KS]), and it became clear afterwards how the formulations of results of "positive" type should be. We hope that this will help to better understand the complete picture of these kind of problems. However, each of these two parts of our paper is self-contained and can be read independently.

In order to present the results of the first part of our article we have to introduce Katznelson's theorem [K1]. In 1974, Katznelson gave an answer to a problem posed in $[\mathrm{B}$, p. 527 , demonstrating that it is impossible to get absolute convergence in Men'shov's theorem. He proved in fact that: "There exists a continuous $2 \pi$-periodic function on the real line that fails to coincide on any set of positive measure with a function whose Fourier series in the trigonometric system is absolutely convergent."

Later on, answering a question posed by Ul'yanov [U1], Olevskii [0] has shown that one can strengthen Katznelson's theorem, obtaining the existence of a continuous function that fails to coincide on any set of positive measure with a function whose Fourier coefficients belong to any class $l^{p}, 1 \leq p<2$.

Motivated by a question posed in GW], Kazarian Ka in 1981 proved that Men'shov's theorem cannot be extended to the class of orthonormal systems (from now on, ONS) which are uniformly bounded and complete: "For any $0<\alpha<1$ there exist a uniformly bounded $L^{2}$-complete ONS $\Phi_{\alpha}=\left\{\phi_{n}\right\}_{n=1}^{\infty}$ of functions on $[0,1]$ and a continuous function $g$ such that the orthogonal expansion of any integrable function $f$ with $|\{x: f(x)=g(x)\}|>\alpha$ in the system $\Phi_{\alpha}$ does not converge uniformly." 
This theorem has recently been improved in GKK]. It was shown that in its formulation one can get that the orthogonal expansion of any integrable function $f$ with $|\{x: f(x)=g(x)\}|>\alpha$ in the system $\Phi_{\alpha}$ does not converge in the $L^{p}$ metric for any $p>2$.

In these theorems the systems which were constructed depended on the number $\alpha$. Now, in our work, we are able to get rid of that condition and, moreover, make the construction in such a way that the resulting functions are continuous. In order to avoid any misunderstanding we recall that a system of elements in a Banach space is called complete if its finite linear combinations are everywhere dense in that space. Also, the notion of orthogonality is always to be considered in connection with the natural inner product of $L^{2}$. In particular, every ONS is made up of elements in $L^{2}$.

Theorem 1.1. There exists an ONS $\Phi=\left\{\phi_{n}\right\}_{n=1}^{\infty}$ of continuous functions defined on $[0,1], \phi_{n}(0)=\phi_{n}(1)=0$, that is complete in all the spaces $L_{[0,1]}^{p}, 1 \leq p<\infty$, which satisfies the uniform estimate

$$
\left|\phi_{n}(x)\right| \leq C, \quad x \in[0,1], n \in \mathbf{N},
$$

and with the following property: for every $m=0,1,2, \ldots$ there exists a function $g_{m} \in C^{m}$ such that for every $f \in L_{[0,1]}$ which coincides with $g_{m}$ on a set of positive measure the Fourier series of $f$ with respect to the system $\Phi$ does not converge in the $L_{[0,1]}^{p}$ metric for any $p>2$.

The above theorem is derived from a more general scheme which has its own interest and is formulated as Theorem 1.6. In $\S 1.2$ the following theorem is also proved.

Theorem 1.5. There exists an ONS $F=\left\{f_{n}\right\}_{n=1}^{\infty}$ of continuous functions defined on $[0,1]$ and such that $f_{n}(0)=f_{n}(1)=0, F$ is complete in all the spaces $L_{[0,1]}^{p}, 1 \leq$ $p<\infty$, and for every $m=0,1,2, \ldots$ there exists a function $g_{m} \in C^{m}$ such that for every $f \in L_{[0,1]}$ which coincides with $g_{m}$ on a set of positive measure, any rearrangement of the Fourier series of $f$ with respect to the system $F$ does not converge in the $L_{[0,1]}^{p}$ metric for any $p>2$.

We would like to mention here that the differences in the formulation of Theorems 1.1 and 1.5 are essential since, as it will become clear later, in Theorem 1.1 we cannot assert the divergence for every rearrangement of the given system and, reciprocally, the assertion of Theorem 1.5 is not true for uniformly bounded complete ONS.

Before presenting our results on what we call the strong $L^{p}$-property, we would like to mention the two papers $[\mathrm{T}]$ and $[\mathrm{P}$. In $[\mathrm{T}]$ a "correction" type lemma was proved for general complete ONS and, as a consequence, it was shown that any complete ONS is a system of representation in the sense of convergence in measure. In $[\mathrm{P}$ it was perhaps for the first time observed that by Fejer's lemma one can easily derive "correction" type lemmas. It was proved there that any complete ONS, under some weak restrictions, becomes, after some fixed rearrangement, a system of representation in the sense of convergence almost everywhere.

Our first result in this direction states that if for $p>2$ we suppose that the ONS $\Phi=\left\{\phi_{n}\right\}_{n=1}^{\infty}$ satisfies the condition

$$
\left\|\phi_{n}\right\|_{p} \leq C_{p} \quad \text { for every } n \in \mathbf{N},
$$


and is complete in $L_{[0,1]}^{p}$, then it turns out that there exists a rearrangement $\sigma$ of the natural numbers so that the system $\Phi_{\sigma}=\left\{\phi_{\sigma(n)}\right\}_{n=1}^{\infty}$ has the strong $L^{p}$-property described in (5) and (6) below. But first we need some notation. Let

$$
S_{\Phi}^{*}(f, x)=\sup _{1 \leq m<\infty}\left|\sum_{k=1}^{m} c_{k}(f) \phi_{k}(x)\right|,
$$

where

$$
c_{k}(f)=\int_{0}^{1} f(x) \phi_{k}(x) d x .
$$

Then the following theorem is true.

Theorem 2.1. Let $\Phi=\left\{\phi_{n}\right\}_{n=1}^{\infty}$ be an ONS complete in the space $L_{[0,1]}^{p}$, for some $p>2$, and assume that (2) holds. Then there exists a rearrangement $\sigma$ of the natural numbers $\mathbf{N}$ such that the system $\Phi_{\sigma}=\left\{\phi_{\sigma(n)}\right\}_{n=1}^{\infty}$ has the following property:

For every $f \in L_{[0,1]}^{p}$ and $\epsilon>0$ there exists a function $f_{\epsilon} \in L_{[0,1]}^{p}$ such that

$$
\sum_{k=1}^{\infty} c_{\sigma(n)}\left(f_{\epsilon}\right) \phi_{\sigma(n)}=f_{\epsilon} \text { in } L_{[0,1]}^{p},
$$

where the coefficients are defined by (4). Moreover,

$$
\left\|S_{\Phi_{\sigma}}^{*}\left(f_{\epsilon}, \cdot\right)\right\|_{p} \leq C_{p}\left\|f_{\epsilon}\right\|_{p} \leq \frac{C_{p}}{\epsilon^{\frac{p-1}{p}}}\|f\|_{p} .
$$

If the complete ONS $\Phi=\left\{\phi_{n}\right\}_{n=1}^{\infty}$ satisfies slightly stronger conditions, we can show that there exists a rearrangement $\sigma$ of the natural numbers $\mathbf{N}$ such that the system $\Phi_{\sigma}=\left\{\phi_{\sigma(n)}\right\}_{n=1}^{\infty}$ has the strong $L^{p}$-property for every $p>2$. Namely, we have

Theorem 2.2. Let $\Phi=\left\{\phi_{n}\right\}_{n=1}^{\infty}$ be an ONS complete in all the spaces $L_{[0,1]}^{p}, 1 \leq$ $p<\infty$, which satisfies (2) for all $p>2$, but not necessarily with $C_{p}$ uniformly bounded. Then there exists a rearrangement $\sigma$ of the natural numbers $\mathbf{N}$ such that the system $\Phi_{\sigma}=\left\{\phi_{\sigma(n)}\right\}_{n=1}^{\infty}$ has the strong $L^{p}$-property for all $p>2$. In fact, for every $2 \leq p<\infty$ and for every $f \in L_{[0,1]}^{p}$ and $\epsilon>0$ there exists a function $f_{\epsilon} \in L_{[0,1]}^{p}$ such that conditions (5) and (6) hold.

Moreover, (7) is true (including the case $p=2$ ), and if $f \in L_{[0,1]}^{p}$ for all $1 \leq p<$ $\infty$ and $\epsilon>0$, then there exists a function $f_{\epsilon} \in \bigcap_{1 \leq r<\infty} L_{[0,1]}^{r}$ such that conditions (5) and (6) are true for all $2<p<\infty$.

Let us make the observation that Theorems 2.1 and 2.2 are no longer true if we do not allow a permutation of the system. This can be easily seen from what was formulated above, namely, from Theorems 1.1 and 1.5.

Every theorem about modification of functions can be accompanied by a result on the existence of universal series. If $M$ is a class of measurable functions and $\left\{f_{n}\right\}_{n=1}^{\infty}$ is a sequence of functions from that class, then a series $\sum_{n=1}^{\infty} f_{n}$ is said to be universal with respect to subseries in that class in the sense of convergence a.e., 
or in some metric, if for each function $f \in M$ there is a subseries

$$
\sum_{k=1}^{\infty} f_{n_{k}}, \quad n_{1}<n_{2}<\cdots
$$

converging a.e., or in the given metric, to $f$. Instead of subseries one also can take rearrangements or subsequences of partial sums of the given series. Obviously one can define universal series for more general objects. In this context, the famous theorem of Riemann on numerical series can be interpreted as the first result about universal series. One of the first results about universal series of functions was proved by Orlicz Or] in 1927; he showed that in the class of all measurable functions there exists a series of functions which is universal with respect to rearrangements in the sense of a.e. convergence. Trigonometric series that are universal with respect to subsequences of partial sums in the sense of convergence almost everywhere were constructed by Men'shov [M3] and Kozlov [Ko]. Orthogonal series universal with respect to rearrangements have been first studied by $\mathrm{Ul}^{\prime}$ yanov [U2].

It can be easily seen that there do not exist universal series with respect to any of the above mentioned means in the sense of convergence in $L_{[0,1]}^{p}, p \geq 2$. We will see, however, that there exist universal series in some weaker sense, as the following two theorems show.

Theorem 2.3. Let $\Phi=\left\{\phi_{n}\right\}_{n=1}^{\infty}$ be an ONS complete in the space $L_{[0,1]}^{p}$, for some $p \geq 2$, and assume that (2) holds. Then there exist a rearrangement $\sigma$ of the natural numbers $\mathbf{N}$ and a series

$$
\sum_{n=1}^{\infty} b_{n} \phi_{\sigma(n)}
$$

with the following properties:

For every $f \in L_{[0,1]}^{p}$ and $\epsilon>0$ there exist a function $f_{\epsilon} \in L_{[0,1]}^{p}$ and a subsequence $\left\{n_{k}\right\}_{k=1}^{\infty}$ such that $\left|\left\{x: f(x) \neq f_{\epsilon}(x)\right\}\right| \leq \epsilon$ and

$$
\sum_{k=1}^{\infty} b_{n_{k}} \phi_{\sigma\left(n_{k}\right)}=f_{\epsilon} \quad \text { in } L_{[0,1]}^{p} ; \quad \sum_{k=1}^{\infty}\left|b_{n_{k}}\right|^{r}<\infty \quad \text { for every } r>2 .
$$

This gives us the existence of universal series with respect to subseries in the sense of $L^{p}$-convergence, $p \geq 2$, after an $\epsilon$-modification of our given function. It also gives precise estimates on the size of the coefficients of the expansion of $f_{\epsilon}$ with respect to the system $\Phi_{\sigma}$.

Theorem 2.4. Let $\Phi=\left\{\phi_{n}\right\}_{n=1}^{\infty}$ be an ONS, complete in all the spaces $L_{[0,1]}^{p}, 1 \leq$ $p<\infty$, and satisfying (2) for all $p<\infty$. Then there exist a rearrangement $\sigma$ of the natural numbers $\mathbf{N}$ and a series (8) such that:

For every $f \in \bigcap_{1 \leq r<\infty} L_{[0,1]}^{r}$ and $\epsilon>0$ there exist a function $f_{\epsilon} \in \bigcap_{1 \leq r<\infty} L_{[0,1]}^{r}$ and a subsequence $\left\{n_{k}\right\}_{k=1}^{\infty}$ such that $\left|\left\{x: f(x) \neq f_{\epsilon}(x)\right\}\right| \leq \epsilon$ and

$$
\begin{gathered}
\sum_{k=1}^{\infty} b_{n_{k}} \phi_{\sigma\left(n_{k}\right)}=f_{\epsilon} \quad \text { in } L_{[0,1]}^{p}, \text { for all } 1 \leq p<\infty ; \\
\sum_{k=1}^{\infty}\left|b_{n_{k}}\right|^{r}<\infty, \quad \text { for every } r>2 .
\end{gathered}
$$


Different constants in the text frequently are denoted by the same letter, with the parameter on which these numbers depend given by an index.

\section{Complete orthonormal systems Without the Strong $L^{p}$-Property}

1.1. Definitions and auxiliary results. Set

$$
s_{n}(x)=\sqrt{2} \sin \pi n x, \quad n \in \mathbf{N}, x \in[0,1],
$$

and, for every $k \in \mathbf{N}$, let us put $\Delta_{k}=\left[1-2^{-k+1}, 1-2^{-k}\right)$. We will say that a function $g$ defined on the semi-interval $[0,1)$ belongs to the class $\hat{C}^{\infty}$ if

$$
\sum_{n=1}^{\infty} n^{m}\left|b_{n}(g)\right|<\infty \quad \text { for every } m \in \mathbf{N},
$$

where

$$
b_{n}(g)=2 \int_{0}^{1} g(t) \sin \pi n t d t .
$$

Denote by $\chi_{\Delta}$ the characteristic function of the interval $\Delta$ and let $I_{\Delta}$ be the increasing affine transformation which maps $\Delta$ onto $[0,1)$. By $I_{\Delta}^{-1}$ we will denote its inverse transformation. The following class of functions is also introduced:

$$
\hat{C}_{\Delta}^{\infty}=\left\{f \circ I_{\Delta}: f \in \hat{C}^{\infty}\right\} .
$$

Let $\Lambda=\left\{\lambda_{\nu}\right\}_{\nu=1}^{\infty}$ be an increasing sequence of natural numbers such that the remaining set of numbers $\mathbf{N} \backslash \Lambda$ is not finite, and denote by $\hat{C}_{\Lambda}^{\infty}$ the class of integrable functions $g$ defined on $[0,1)$ such that

$$
\sum_{\substack{n=1 \\ n \neq \lambda_{\nu}}}^{\infty} n^{m}\left|b_{n}(g)\right|<\infty \quad \text { for every } m \in \mathbf{N}
$$

where the coefficients $b_{n}(g)$ are defined by (9). Similarly as above, we denote

$$
\hat{C}_{\Delta, \Lambda}^{\infty}=\left\{f \circ I_{\Delta}: f \in \hat{C}_{\Lambda}^{\infty}\right\}
$$

For the construction of uniformly bounded complete ONS we will use a well known method which is formulated in the following lemma (see GKK]).

Lemma 1.2. Let $B$ be a closed subspace of $L_{[0,1]}^{2}$ which can be represented as a direct sum of two closed orthogonal infinite dimensional subspaces of $L_{[0,1]}^{2}, B=$ $B_{1} \oplus B_{2}$, such that $B_{1}$ has an orthonormal basis $G_{1}=\left\{g_{k}^{1}\right\}_{k=1}^{\infty}$ consisting of functions from the space $L_{[0,1]}^{\infty}$ and $B_{2}$ has a uniformly bounded orthonormal basis $G_{2}=\left\{g_{k}^{2}\right\}_{k=1}^{\infty}$; that is,

$$
\left\|g_{k}^{2}\right\|_{\infty} \leq C<\infty, \quad k \in \mathbf{N} .
$$

Then $B$ has a uniformly bounded orthonormal basis $\Phi=\left\{\Phi_{k}\right\}_{k=1}^{\infty}$, and there exists an increasing sequence of natural numbers $\left\{n_{k}\right\}_{k=1}^{\infty}$ such that

$$
g_{k}^{1}=\sum_{m=n_{k}}^{n_{k+1}-1} a_{m} \Phi_{m}
$$

Moreover, each element of $\Phi$ can be represented as a finite linear combination of some elements of $G_{1}$ and $G_{2}$. 
In GKK this lemma is formulated without the last conclusion. We have added this observation because we will need it in our proofs. One can easily see that it is true by examining of the proof of Lemma 1.2 in GKK].

For proving the main result of part 1 of this article we need the following modification of a result by A. Zygmund ([Z], Vol. 1, p. 203).

Lemma 1.3. Let $\Sigma=\left\{E_{m}\right\}_{m=1}^{\infty}$ be a sequence of sets in $[0,1]$ of positive measure satisfying

$$
\left|E_{m} \Delta E\right| \rightarrow 0 \quad \text { when } m \rightarrow \infty, \quad \text { with }|E|>0,
$$

where $\left|E_{m} \Delta E\right|=\left(E_{m} \backslash E\right) \cup\left(E \backslash E_{m}\right)$, and consider fixed numbers $C>1$ and $q>1$. Then there exist integers $N_{0}=N_{0}(E, C, q)$ and $M_{0}=M_{0}(E, C, q, \Sigma)$ such that for any real trigonometric polynomial of the form

$$
P(x)=\sum_{j \geq 1}\left(a_{j} \cos 2 \pi n_{j} x+b_{j} \sin 2 \pi n_{j} x\right),
$$

with $n_{1} \geq N_{0}$ and $n_{j+1} / n_{j}>q>1$, and for $m \geq M_{0}$, we have

$$
C^{-1}\left|E_{m}\right| \frac{1}{2} \sum\left(a_{j}^{2}+b_{j}^{2}\right) \leq \int_{E_{m}} P^{2}(x) d x \leq C\left|E_{m}\right| \frac{1}{2} \sum\left(a_{j}^{2}+b_{j}^{2}\right) .
$$

Proof. Writing the polynomial $P$ in the complex form $\sum_{\nu \in \mathbf{Z}} c_{\nu} \exp \left(2 \pi i n_{\nu} x\right)$, with $n_{-\nu}=-n_{\nu}$, we have

$$
\begin{gathered}
\int_{E_{m}} P^{2}(x) d x=\int_{E_{m}}\left[\sum c_{\nu} \exp \left(2 \pi i n_{\nu} x\right)\right]\left[\sum \bar{c}_{\nu} \exp \left(-2 \pi i n_{\nu} x\right)\right] d x \\
=\left|E_{m}\right| \sum\left|c_{\nu}\right|^{2}+\sum_{\mu \neq \nu} c_{\mu} \bar{c}_{\nu} \int_{E_{m}} \exp \left[2 \pi i\left(n_{\mu}-n_{\nu}\right) x\right] d x .
\end{gathered}
$$

Denote by $\gamma_{k}^{(m)}$ (respectively by $\gamma_{k}$ ) the complex Fourier coefficients of the characteristic function of $E_{m}$ (respectively of $E$ ). Then the absolute value of the last sum can be estimated from above by

$$
\left[\sum_{\mu, \nu}\left|c_{\mu} c_{\nu}\right|^{2}\right]^{\frac{1}{2}}\left(\sum_{\mu \neq \nu}\left|\gamma_{n_{\mu}-n_{\nu}}^{(m)}\right|^{2}\right)^{\frac{1}{2}}=\left(\sum_{\mu}\left|c_{\mu}\right|^{2}\right)\left(\left(\sum_{\mu \neq \nu}\left|\gamma_{n_{\mu}-n_{\nu}}^{(m)}\right|^{2}\right)^{\frac{1}{2}} .\right.
$$

It is well known that there exists a number $B_{q}$ such that no integer $n$ can be represented more than $B_{q}$ times in the form $n_{\mu}-n_{\nu}$ with $\mu \neq \nu$ if $\left\{n_{j}\right\}$ is as above ([Z], Vol. 1, pp. 203-204). Thus, the last factor in the right-hand side of (11) does not exceed $\left[2 B_{q} \sum_{N_{0}}^{\infty}\left|\gamma_{k}^{(m)}\right|^{2}\right]^{\frac{1}{2}}$, where $N_{0}$ is smaller than the least integer representable in the form $n_{\mu}-n_{\nu}$ with $1 \leq \nu<\mu$. Now, if this $N_{0}$ is sufficiently large (depending on $E, \epsilon>0$ and $q$ ), we have also

$$
\left[2 B_{q} \sum_{N_{0}}^{\infty}\left|\gamma_{k}\right|^{2}\right]^{\frac{1}{2}}<\epsilon|E|
$$

On the other hand, the condition $\left|E \Delta E_{m}\right|=\int\left|\chi_{E}-\chi_{E_{m}}\right|^{2} \rightarrow 0$ says that $\left|E_{m}\right| \rightarrow$ $|E|$ and $\sum\left|\gamma_{k}-\gamma_{k}^{(m)}\right|^{2} \rightarrow 0$ as $m \rightarrow \infty$ and, therefore, for $M_{0}$ large enough and $m>M_{0}$, the right hand side of (11) is majorized by $\left(\sum_{\mu}\left|c_{\mu}\right|^{2}\right)\left|E_{m}\right| \epsilon$. It suffices then to take $\epsilon=C-1$ and $\epsilon=1-\frac{1}{C}$ to obtain the inequalities stated in the lemma. 
1.2. Complete orthonormal systems without the strong $L^{p}$-property after any rearrangement. In this section we will give a proof of Theorem 1.5 stated in the introduction. Define, for $k, n \in \mathbf{N}$,

$$
t_{n}^{k}(x)= \begin{cases}2^{\frac{k+1}{2}} s_{n}\left[I_{\Delta_{k}}(x)\right] & \text { for } x \in \Delta_{k} \\ 0 & \text { otherwise on }[0,1]\end{cases}
$$

It is clear that all these functions are continuous on $[0,1]$ and that $\mathbf{T}=\left\{t_{n}^{k}, k, n \in\right.$ $\mathbf{N}\}$ is a complete ONS. It is rather obvious that $\mathbf{T}$ is complete in all the spaces $L_{[0,1]}^{p}, 1 \leq p<\infty$. Define

$$
s_{n}^{k, i}(x)=\frac{1}{\sqrt{2}}\left(t_{n}^{k}(x)+(-1)^{i-1} t_{k}^{n+1}(x)\right) \quad(n \geq k ; k=1,2, \ldots ; i=1,2) .
$$

From the completeness and orthonormality of the system (12) we immediately deduce that the set of functions $\left\{s_{n}^{k, i}, n \geq k ; k=1,2, \ldots ; i=1,2\right\}$ also forms a complete ONS.

Lemma 1.4. Let $f \in L_{[0,1]}^{p}$ for $p>2$, and assume that $f \chi_{\Delta_{k}} \notin \hat{C}_{\Delta_{k}}^{\infty}$ for some $k \in \mathbf{N}$. Then,

$$
\varlimsup_{n \rightarrow \infty}\left\|c_{n}^{k, i} s_{n}^{k, i}\right\|_{L_{\Delta_{n+1}}^{p}}=+\infty
$$

where

$$
c_{n}^{k, i}=\int_{0}^{1} f(t) s_{n}^{k, i}(t) d t .
$$

Proof. Set $g=\left(f \chi_{\Delta_{k}}\right) \circ I_{\Delta_{k}}^{-1}$. Since $f \chi_{\Delta_{k}} \notin \hat{C}_{\Delta_{k}}^{\infty}$ for some $k \in \mathbf{N}$, then there exists $m \in \mathbf{N}$ for which

$$
\sum_{n=1}^{\infty} n^{m}\left|b_{n}(g)\right|=\infty
$$

where $b_{n}(g)$ are the Fourier coefficients of the function $g$ with respect to the system $S=\left\{s_{n}\right\}_{n=1}^{\infty}$. By (12) and (13) we get

$$
\begin{aligned}
c_{n}^{k, i} & =\frac{1}{\sqrt{2}} \int_{\Delta_{k}} f(x) t_{n}^{k}(x) d x+(-1)^{i-1} 2^{\frac{n+2}{2}} \int_{\Delta_{n+1}} f(x) s_{k}\left[I_{\Delta_{n+1}}(x)\right] d x \\
& =b_{n}^{k}+(-1)^{i-1} b_{k}^{n+1}
\end{aligned}
$$

Also

$$
\left\|s_{n}^{k, i}\right\|_{L_{\Delta_{n+1}}^{p}}=\alpha_{p, 1} 2^{n\left(\frac{1}{2}-\frac{1}{p}\right)}, \quad n \geq k
$$

where $\alpha_{p, k}>0$ is independent of $n$. We obtain (14) by (15)-(18) and the following claim (which is an easy consequence of Hölder's inequality).

Claim. For any $k, m \in \mathbf{N}$, with the above notation,

$$
\sum_{n=k}^{\infty} n^{m}\left|b_{k}^{n+1}\right| \leq C\|g\|_{p} \sum_{n=k}^{\infty} n^{m} 2^{\frac{n+1}{2}-\frac{n+1}{q}}<+\infty
$$

where $C>0$ is an absolute constant and $q=\frac{p}{p-1}<2$. 
This finishes the proof of the lemma. In order to conclude the proof of Theorem 1.5 it suffices to take $F$ as any enumeration of the system $\left\{s_{n}^{k, i}, n \geq k ; k=\right.$ $1,2, \ldots ; i=1,2\}$ and then use Lemma 1.8 below (in fact a weaker version of it, since we can let $\Lambda=\emptyset$ here). If $\left\{f_{m}\right\}$ are the functions constructed in that lemma, then our smooth functions in Theorem 1.5 are $g_{m}=\sum_{k}\left(f_{m} \circ I_{\Delta_{k}}\right) \chi_{\Delta_{k}}$.

1.3. Uniformly bounded complete orthonormal systems without the strong $L^{p}$-property. The following theorem is proved to make more transparent the existence of systems that was asserted in Theorem 1.1.

Theorem 1.6. Let $\Lambda=\left\{\lambda_{\nu}\right\}_{\nu=1}^{\infty}$ be an increasing sequence of natural numbers such that the remaining set of numbers $\mathbf{N} \backslash \Lambda$ is not finite, and let $k_{0} \in \mathbf{N}$ be given. Then there exists a uniformly bounded ONS $\Phi_{\Lambda}^{\left(k_{0}\right)}=\left\{\phi_{n}\right\}_{n=1}^{\infty}$ of continuous functions defined on the segment $[0,1], \phi_{n}(0)=\phi_{n}(1)=0$,

$$
\left|\phi_{n}(x)\right| \leq C, \quad x \in[0,1], n \in \mathbf{N},
$$

that is complete in the spaces $L_{[0,1]}^{p}, 1 \leq p<\infty$, and with the property that for every function $f \in L_{[0,1]}$ such that

$$
f \chi_{\Delta_{k}} \notin \hat{C}_{\Delta_{k}}^{\infty} \quad \text { for some } k \neq k_{0} \quad \text { or } \quad f \chi_{\Delta_{k_{0}}} \notin \hat{C}_{\Delta_{k_{0}}, \Lambda}^{\infty}
$$

its Fourier series with respect to the system $\Phi_{\Lambda}^{\left(k_{0}\right)}$ diverges in any metric $L_{[0,1]}^{p}$, $p>2$.

Proof. The construction of the system $\Phi_{\Lambda}^{\left(k_{0}\right)}$.

Let $\left\{\hat{t}_{n}^{k_{0}}\right\}_{n \in \mathbf{N}}$ be any enumeration of the subset $\left\{t_{n}^{k_{0}} ; \quad n \in \mathbf{N} \backslash \Lambda\right\}$. Recall that the functions $t_{n}^{k}$ were defined in the previous section. For simplicity let us write

$$
\hat{t}_{n}^{k}=t_{n}^{k} \quad \text { when } k \neq k_{0} \text { and } k \in \mathbf{N} .
$$

Define

$$
\hat{s}_{n}^{k, i}(x)=\frac{1}{\sqrt{2}}\left(\hat{t}_{n}^{k}(x)+(-1)^{i-1} \hat{t}_{k}^{n+1}(x)\right) \quad(n \geq k ; k=1,2, \ldots ; i=1,2) .
$$

It is clear again that these new functions are continuous on $[0,1]$ and that the family $\left\{\hat{s}_{n}^{k, i}, n \geq k ; k \in \mathbf{N} ; i=1,2\right\} \cup\left\{t_{\lambda_{\nu}}^{k_{0}}\right\}_{\nu=1}^{\infty}$ is a complete ONS.

The proof of the analogue of Lemma 1.4 for the system $\left\{\hat{s}_{n}^{k, i}, n \geq k ; k \in \mathbf{N} ; i=\right.$ $1,2\}$, which only requires some obvious modifications for the case $k=k_{0}$, is completely similar. Hence, taking as $G_{1}$ some enumeration of the set of functions

$$
\left\{\hat{s}_{n}^{k, i}, n \geq k ; k \in \mathbf{N} ; i=1,2\right\}
$$

and letting the ONS $\left\{\hat{t}_{\lambda_{\nu}}^{k_{0}}\right\}_{\nu=1}^{\infty}$ stay for the uniformly bounded system $G_{2}$, we easily obtain from Lemma 1.7 that the uniformly bounded complete ONS constructed in this way has all desired properties of Theorem 1.6. The new system will be denoted by $\Phi_{\Lambda}^{\left(k_{0}\right)}$.

In order to continue now with the proof of Theorem 1.1, we have to prove two additional lemmas. The first of them establishes a direct relation between the lacunarity of the Fourier series of a function, its modulus of continuity, and absolute summability. 
Lemma 1.7. Let a function $g \in L_{[0,1]}^{2}$ be given in the form

$$
g(x)=\sum_{-\infty}^{\infty} c_{\nu} \exp \left(2 \pi i n_{\nu} x\right), \quad \text { where }-n_{\nu}=n_{-\nu},
$$

with $n_{j+1} / n_{j}>q>1$, and suppose that $g$ coincides on a set $E$ of positive measure with a continuous 1-periodic function $f$ with modulus of continuity $\omega(\delta)$. Then, if

$$
\sum_{1}^{\infty} \omega\left(\frac{1}{2} n_{\nu}^{-1}\right)<\infty
$$

the series expansion of $g$ is absolutely summable; that is, $\left\{c_{\nu}\right\} \in l^{1}$.

Proof. Suppose that the function $g$ is periodically continued on the real line with a period equal to 1 . Then, for $0<h<1$ we have

$$
g(x+h)-g(x-h)=-2 i \sum \sin \left(2 \pi h n_{\nu}\right) c_{\nu} \exp \left(2 \pi i n_{\nu} x\right) .
$$

Denote

$$
E_{\nu}=\left\{x \in E: x \pm \frac{1}{4} n_{\nu}^{-1} \in E\right\}
$$

and let $\chi$ be the characteristic function of the set $E$. Then, from the fact that

$$
\int|\chi(x+t)-\chi(x)| d x \rightarrow 0 \quad \text { for } t \rightarrow 0,
$$

we get that

$$
\left|E \Delta E_{\nu}\right| \rightarrow 0 \quad \text { when } \nu \rightarrow \infty
$$

Hence, by Lemma 1.3, putting $C=2$, we have that there exist integers $N_{0}$ and $M_{0}$ such that when $\nu>M_{0}$

$$
\frac{1}{4}\left|E_{\nu}\right| \sum_{n_{j} \geq N_{0}}\left|c_{j}\right|^{2} \leq \int_{E_{\nu}}|g(x)|^{2} d x
$$

Therefore, by (20),

$$
\int_{E_{\nu}}|g(x+h)-g(x-h)|^{2} d x \geq\left|E_{\nu}\right| \sum_{n_{j} \geq N_{0}}\left|c_{j}\right|^{2} \sin ^{2}\left(2 \pi n_{j} h\right) .
$$

Setting $h=\frac{1}{4} n_{\nu}^{-1}$, we have, from the definition of $E_{\nu}$ and our hypothesis on $f$,

$$
\int_{E_{\nu}}|g(x+h)-g(x-h)|^{2} d x=\int_{E_{\nu}}|f(x+h)-f(x-h)|^{2} d x .
$$

Thus the left-hand side of (21) does not exceed $\omega\left(\frac{1}{2} n_{\nu}^{-1}\right)^{2}\left|E_{\nu}\right|$ for $\nu>M_{0}$, and hence, for $n_{\nu} \geq N_{0}$ and $\nu>M_{0}$, we obtain $\left|c_{\nu}\right| \leq \omega\left(\frac{1}{2} n_{\nu}^{-1}\right)$.

Lemma 1.8. There exists an increasing sequence of natural numbers $\Lambda=\left\{\lambda_{\nu}\right\}_{\nu=1}^{\infty}$ such that for every integer $m=0,1,2, \ldots$ one can construct a function $f_{m} \in C^{m}$ satisfying

$$
\frac{d^{j}}{d x^{j}} f_{m}(0)=\frac{d^{j}}{d x^{j}} f_{m}(1)=0, \quad 0 \leq j \leq m,
$$


and, if $g \in L_{[0,1]}$ coincides with $f_{m}$ on a set of positive measure, then

$$
\sum_{\substack{n=1 \\ n \neq \lambda_{\nu}}}^{\infty} n^{m}\left|b_{n}(g)\right|=\infty
$$

Proof. Let

$$
\lambda_{\nu}=2^{2^{\nu}} \quad \text { for } \nu \in \mathbf{N},
$$

and let us construct our function for the case $m=0$. As in the proof of Katznelson's theorem, we use the so-called Rudin-Shapiro sequence $\left\{\epsilon_{j}\right\}_{0}^{\infty}, \epsilon_{j}= \pm 1$, which has the following property:

For all positive integers $n, m \in \mathbf{N}$

$$
\left\|\sum_{j=n+1}^{n+m} \epsilon_{j} e^{\pi i j x}\right\|_{C_{[-1,1]}} \leq 5 \sqrt{m}
$$

(see [K2, pp. 33-34). Define for $x \geq 0$

$$
\Phi(x)=\left\{\begin{array}{l}
\epsilon_{j}, \quad \text { if } \quad j \leq x \leq j+\frac{1}{2}, \\
\text { linear for } j+\frac{1}{2} \leq x \leq j+1,
\end{array}\right.
$$

and

$$
\Phi_{k}(x)=\Phi\left(\frac{8^{k} x}{2}\right) .
$$

Let $k_{m}=m$ ! and, for $m \in \mathbf{N}$, set

$$
\Phi_{m}^{*}(x)=\left\{\begin{array}{l}
0 \quad \text { for } x=0, x=1, \\
\Phi_{k_{m}}(x) \quad \text { if } x \in\left[8^{-k_{m}}, 1-8^{-k_{m}}\right], \\
\text { linear for } 0 \leq x \leq 8^{-k_{m}} \text { and } 1-8^{-k_{m}} \leq x \leq 1 .
\end{array}\right.
$$

We now define our function on the segment $[0,1]$ by the following formula:

$$
f_{0}(x)=\sum_{m=1}^{\infty} k_{m}^{-1} \Phi_{m}^{*}(x),
$$

and we extend it on $[-1,0]$ to be an odd function. By an obvious modification of Katznelson's original proof we arrive at the claim that if a function $g$ is the sum of an absolutely convergent series with respect to the system $\mathbf{S}=\left\{s_{n}(x)\right\}_{n=1}^{\infty}$, then the set $\left\{x: f_{0}(x)=g(x)\right\}$ has measure zero. On the other hand, by an easy calculation we deduce from (24) - (27) that the modulus of continuity of $f_{0}$ satisfies

$$
\omega\left(f_{0}, \delta\right) \leq C \frac{1}{\log \delta^{-1}} .
$$

In particular, from Lemma 1.7 and (23) we obtain $\sum_{n=1}^{\infty}\left|b_{\lambda_{\nu}}(g)\right|<\infty$, and by the above remark, we get (22).

For $m \geq 1$ we obtain the function $f_{m}$ by integrating $f_{m-1}$ and subtracting afterwards $a_{m} \phi_{m}(x)$, where $\phi_{m}$ is an $(m+2)$-times continuously differentiable function, $a_{m}=\int_{0}^{1} \phi_{m-1}(t) d t$ and

$$
\frac{d^{j}}{d x^{j}} \phi_{m}(0)=\frac{d^{j}}{d x^{j}} \phi_{m}(1)=0 \quad(0 \leq j \leq m-1) ;
$$




$$
\frac{d^{m}}{d x^{m}} \phi_{m}(0)=0 ; \quad \frac{d^{m}}{d x^{m}} \phi_{m}(1)=1
$$

If two differentiable functions coincide on a set of positive measure, then they coincide on the closure of that set. Hence, their derivatives will coincide on a set that contains the points of density of the given set, and that again has the same measure.

Proof of Theorem 1.1. It is an easy consequence of Theorem 1.6 and Lemma 1.8. We let $\Lambda=\left\{\lambda_{\nu}\right\}_{\nu=1}^{\infty}$ be any sequence of increasing natural numbers for which the assertion of Lemma 1.8 is true, and then we take $\Phi_{\Lambda}^{(1)}=\left\{\phi_{n}\right\}_{n=1}^{\infty}$ to be our system. Putting

$$
g_{m}(x)=\alpha_{k}\left\|f_{m} \circ I_{\Delta_{k}}\right\|_{C^{m}}^{-1} f_{m}\left(I_{\Delta_{k}}(x)\right) \quad \text { for } x \in \Delta_{k}, k \in \mathbf{N},
$$

where $\left\{\alpha_{k}\right\}_{k=1}^{\infty}$ is any sequence of positive numbers tending to zero, we finish the proof.

\section{The STRONG $L^{p}$-PROPERTY FOR ORTHOGONAL SYSTEMS}

2.1. Definitions and auxiliary results. Let us start by recalling the definition of independent functions. This will help to simplify some calculations.

Definition 2.5. Two functions $f$ and $g$ are called independent if for any given numbers $a, b, c, d$ with $a<b$ and $c<d$ the following equality is true:

$$
\begin{gathered}
|\{x \in[0,1]: a \leq f(x)<b, c \leq g(x)<d\}| \\
=|\{x \in[0,1]: a \leq f(x)<b\}||\{x \in[0,1]: c \leq g(x)<d\}| .
\end{gathered}
$$

From this definition one can easily verify that if two given integrable functions $f$ and $g$ are independent, then

$$
\int_{0}^{1} f(x) g(x) d x=\int_{0}^{1} f(x) d x \int_{0}^{1} g(x) d x .
$$

Let us mention another easy consequence of the definition. If the functions $f$ and $g$ are independent, then $|f|$ and $|g|$ are also independent, and, moreover, the functions $|f|^{\alpha}$ and $|g|^{\beta}$, for any positive numbers $\alpha, \beta$, have the same property.

One of the main tools in the Men'shov type constructions is the following simple observation (see [Z], p. 49)

Lemma 2.6 (Fejer). Let $\phi \in L_{[0,1]}^{p}, 1 \leq p \leq \infty$, be a 1-periodic function on the real line $\mathbf{R}$. Then for every $f \in L_{[0,1]}^{p^{\prime}}$, where $p^{\prime}=1$ or $\infty$ if, respectively, $p=\infty$ or 1 , and $p^{\prime}=\frac{p}{p-1}$ if $1<p<\infty$, the following equality holds:

$$
\lim _{n \rightarrow \infty} \int_{0}^{1} f(x) \phi(n x) d x=\int_{0}^{1} f(x) d x \int_{0}^{1} \phi(x) d x .
$$

For every $0<h<1$ we define

$$
J_{h}(x)= \begin{cases}-\frac{1-h}{h} & \text { for } x \in[0, h], \\ 1 & \text { for } x \in(h, 1] .\end{cases}
$$

We need Garsia's result about permutations of given numbers (G], pp. 93-97), which is one of the crucial points in the proof of Theorem 2.2. 
Theorem 2.7 (Garsia). Let $\left\{\alpha_{j}\right\}_{j=1}^{n}$ be a sequence of real numbers. Then, if $1 \leq$ $p<\infty$,

$$
\frac{1}{n !} \sum_{\sigma} \max _{1 \leq \nu \leq n}\left|\alpha_{\sigma_{1}}+\alpha_{\sigma_{2}}+\cdots+\alpha_{\sigma_{\nu}}\right|^{p} \leq C_{p}^{p}\left[\left(\sum_{j=1}^{n} \alpha_{j}^{2}\right)^{\frac{1}{2}}+\left|\sum_{j=1}^{n} \alpha_{j}\right|\right]^{p},
$$

where the sum on the left is taken over all permutations $\sigma$ of the numbers $\{1,2, \ldots$, $n\}$, and $C_{p}>0$ depends only on $p$.

2.2 Modification of functions. We are going to present two lemmas which will be needed in order to prove Theorems 2.1, 2.2 and 2.3, 2.4 stated in the introduction. Lemma 2.9 uses the same hypotheses as Lemma 2.8 but the conclusion is stronger. It is, though, technically more complicated and for that reason we have found it more convenient to separate the result into these two parts. Moreover, for the proof of Theorems 2.1 and 2.2 one only needs Lemma 2.8, whereas for Theorems 2.3 and 2.4 one needs the full strength of Lemma 2.9.

Lemma 2.8. Let $\Phi=\left\{\phi_{n}\right\}_{n=1}^{\infty}$ be an ONS complete in the space $L_{[0,1]}^{p}$, for some $p>2$, and assume that (2) holds. Then, for any given function $f \in L_{[0,1]}^{p}$, every positive numbers $0<\delta<1$, every $\eta>0$ and any natural number $N_{0}>2$ there exist a function $\hat{f} \in L_{[0,1]}^{p}$ and a polynomial (with respect to the system $\left.\Phi\right) H(x)=$ $\sum_{N_{0}}^{N_{1}} a_{k} \phi_{k}(x)$, such that

$$
\begin{array}{ll}
\text { a) } & |\{x: f(x)=\hat{f}(x)\}| \geq 1-\delta ; \\
\text { b) } & \|\hat{f}\|_{p}<2 \delta^{-\frac{1}{p^{p}}}\|f\|_{p} ; \\
\text { c) } & \|\hat{f}-H\|_{p}<\eta .
\end{array}
$$

Moreover, there exists a rearrangement $\pi_{0}$ of the numbers $N_{0}, N_{0}+1, \ldots, N_{1}$ such that

$$
\text { d) } \quad\left\|\max _{N_{0} \leq m \leq N_{1}}\left|\sum_{k=N_{0}}^{m} a_{\pi_{0}(k)} \phi_{\pi_{0}(k)}(\cdot)\right|\right\|_{p} \leq C_{p} \delta^{-\frac{1}{p^{\prime}}}\|f\|_{p},
$$

where $C_{p}>0$ depends only on $p$.

Proof. Without loss in generality we can suppose that

$$
\eta<\|f\|_{2} .
$$

Let $\Delta_{j}^{i}=\left(\frac{i-1}{2^{j}}, \frac{i}{2^{j}}\right), 1 \leq i \leq 2^{j}, j \in \mathbf{N}$. There exists a function of the form

$$
g(x)=\sum_{i=1}^{2^{n}} \gamma_{i} \chi_{\Delta_{n}^{i}} \quad \text { such that }\|f-g\|_{p}<\frac{\eta}{2},
$$

Let

$$
\eta_{1}=\frac{\eta}{12 C_{p} N_{0}} .
$$

Applying Fejer's lemma with $f=g \cdot \phi_{k}$ and $\phi=J_{\delta}$, and using the fact that $\int_{0}^{1} J_{\delta}=0$, we can find a natural number

$$
s>n
$$


such that the following inequality holds:

$$
\left|b_{k}\right|=\left|\int_{0}^{1} g(x) \phi_{k}(x) J_{\delta}\left(2^{s} x\right) d x\right|<\eta_{1} \quad \text { for every } 1 \leq k \leq N_{0} .
$$

It can easily be seen by (30), (33) and (35) that the functions $g$ and

$$
\hat{g}(x)=g(x) J_{\delta}\left(2^{s} x\right)
$$

are independent. From the completeness of the system $\Phi$ in the space $L_{[0,1]}^{p}$ we can find a polynomial with respect to the system $\Phi$,

$$
P(x)=\sum_{k=1}^{N_{1}} a_{k} \phi_{k}(x)
$$

such that

$$
\|\hat{g}-P\|_{p}<\frac{\eta_{1}}{C_{p}}
$$

By (36), (38) and (39) we obtain

$$
\left|a_{k}\right| \leq\left|b_{k}\right|+\left|b_{k}-a_{k}\right| \leq \eta_{1}+\left|\int_{0}^{1}[\hat{g}(x)-P(x)] \phi_{k}(x) d x\right| \leq 2 \eta_{1} .
$$

Hence, denoting

$$
H(x)=\sum_{N_{0}}^{N_{1}} a_{k} \phi_{k}(x)
$$

by (38), (39), (2) and (34) we deduce that

$$
\|\hat{g}-H\|_{p} \leq\|\hat{g}-P\|_{p}+2 N_{0} C_{p} \eta_{1} \leq \frac{1}{4} \eta .
$$

Now, putting

$$
\hat{f}=f-g+\hat{g}
$$

from (33) and (41) we obtain that for the function $\hat{f}$ and the polynomial $H$ defined by (41) condition c) of Lemma 2.8 holds.

Condition a) of Lemma 2.8 follows easily from the definition of the function $J_{h}$ (see (30)) and by (37), (42).

From the fact that the function $g$ (see $(33)$ ) is constant on the dyadic intervals of length $2^{-n}$, and from (35) and (37), we deduce that the functions $g$ and $\hat{g}$ are independent. Hence, using the properties of independent functions mentioned in section 2.1 , we get

$$
\int_{0}^{1}|\hat{g}(x)|^{p} d x=\int_{0}^{1}\left|J_{\delta}\left(2^{s} x\right)\right|^{p} d x \int_{0}^{1}|g(x)|^{p} d x \leq \delta^{1-p} \int_{0}^{1}|g(x)|^{p} d x .
$$

Thus, condition b) follows immediately from (42), (33), (32) and (43).

For the proof of condition d) we use the following inequality, which we get from (40), (41), Parseval's equality and the independence of the functions $g$ and $\hat{g}$ : 


$$
\begin{gathered}
\sum_{k=N_{0}}^{N_{1}} a_{k}^{2}=\int_{0}^{1}|H(x)|^{2} d x \leq 2 \int_{0}^{1}|\hat{g}(x)|^{2} d x+\frac{\eta^{2}}{8} \\
\leq \frac{2}{\delta} \int_{0}^{1}|g(x)|^{2} d x+\frac{\eta^{2}}{8} \leq \frac{3}{\delta} \int_{0}^{1}|g(x)|^{2} d x .
\end{gathered}
$$

The last inequality follows from the fact that $\eta<2\|g\|_{2}$, which in turn can be easily obtained from (32) and (33).

By Garsia's theorem we have

$$
\begin{aligned}
& \frac{1}{\left(N_{1}-N_{0}+1\right) !} \sum_{\sigma} \int_{0}^{1} \max _{N_{0} \leq m \leq N_{1}}\left|\sum_{k=N_{0}}^{m} a_{\sigma_{k}} \phi_{\sigma_{k}}(x)\right|^{p} d x \\
\leq & C_{p}^{p} \int_{0}^{1}\left\{\left|\sum_{k=N_{0}}^{N_{1}} a_{k} \phi_{k}(x)\right|+\left[\sum_{k=N_{0}}^{N_{1}}\left[a_{k} \phi_{k}(x)\right]^{2}\right]^{\frac{1}{2}}\right\}^{p} d x .
\end{aligned}
$$

Thus, by (2) and the triangle inequality in $L^{\frac{p}{2}}$ we obtain immediately that there exists a rearrangement $\pi_{0}$ of the numbers $N_{0}, N_{0}+1, \ldots, N_{1}$ such that

$$
\left\|\max _{N_{0} \leq m \leq N_{1}}\left|\sum_{k=N_{0}}^{m} a_{\pi_{0}(k)} \phi_{\pi_{0}(k)}(\cdot)\right|\right\|_{p} \leq C_{p}\left[\|H-\hat{g}\|_{p}+\|\hat{g}\|_{p}+\left(\sum_{k=N_{0}}^{N_{1}} a_{k}^{2}\right)^{\frac{1}{2}}\right] .
$$

Now, (45), (41), (42), (44) and (32) gives us condition d).

Lemma 2.9. Under the same hypotheses of Lemma 2.8, and given $\epsilon>0$, there exist a function $\hat{f} \in L_{[0,1]}^{p}$ and a polynomial with respect to the system $\Phi, H(x)=$ $\sum_{N_{0}}^{N_{1}} a_{k} \phi_{k}(x)$, satisfying conditions a), b), c) and d) of that lemma, as well as the estimate

$$
\left.c^{*}\right) \quad \sum_{N_{0}}^{N_{1}}\left|a_{k}\right|^{2+\epsilon}<\epsilon .
$$

Proof. We will repeat the proof of the previous lemma with some appropriate modifications. In this case we write

$$
g(x)=\sum_{i=1}^{2^{n}} \gamma_{i} \chi_{\Delta_{n}^{i}}=\sum_{i=1}^{2^{n}} g_{i}(x) \quad \text { for which }\|f-g\|_{p}<\frac{\eta}{2},
$$

and add the new condition that

$$
\left\|g_{i}\right\|_{2}^{2}<\frac{\delta}{3} \epsilon_{1}^{2}, \quad \text { for every } 1 \leq i \leq 2^{n},
$$

where $\epsilon_{1}$ satisfies the inequality

$$
\epsilon_{1}^{\epsilon} \cdot \frac{9}{\delta^{2}} \int_{0}^{1}|f(x)|^{2} d x<\epsilon
$$

Now, we define the number $\eta_{1}$ as

$$
\eta_{1}=\frac{\eta}{12 C_{p} 2^{n} N_{0}}
$$


We have to apply Fejer's lemma for every function $g_{i}, 1 \leq i \leq 2^{n}$, for a number $N_{0 i}$ which has to be defined according to what happened in the previous step, and for the number

$$
\eta_{i}=\frac{\eta}{12 C_{p} 2^{n} N_{0 i}}
$$

In this way we find natural numbers $s_{i}>n$ such that

$$
\left|b_{k i}\right|=\left|\int_{0}^{1} g_{i}(x) \phi_{k}(x) J_{\delta}\left(2^{s_{i}} x\right) d x\right|<\eta_{i} \quad \text { for every } 1 \leq k \leq N_{0 i} .
$$

Then, we observe that for every $i, 1 \leq i \leq 2^{n}$, the functions $g_{i}$ and

$$
\hat{g}_{i}(x)=g_{i}(x) J_{\delta}\left(2_{i}^{s} x\right)
$$

are independent. From the completeness of the system $\Phi$ in the space $L_{[0,1]}^{p}$ we can find polynomials

$$
P_{i}(x)=\sum_{k=1}^{N_{0 i+1}} a_{k i} \phi_{k}(x)
$$

such that

$$
\left\|\hat{g}_{i}-P_{i}\right\|_{p}<\frac{\eta_{i}}{C_{p}}
$$

By the same argument as in the proof of Lemma 2.8 we see that

$$
\left|a_{k i}\right| \leq\left|b_{k i}\right|+\left|b_{k i}-a_{k i}\right| \leq \eta_{1}+\left|\int_{0}^{1}\left[\hat{g}_{i}(x)-P_{i}(x)\right] \phi_{k}(x) d x\right| \leq 2 \eta_{i} .
$$

Hence, denoting

$$
H_{i}(x)=\sum_{N_{0 i}}^{N_{0 i+1}} a_{k i} \phi_{k}(x),
$$

we deduce that

$$
\left\|\hat{g}_{i}-H_{i}\right\|_{p} \leq\left\|\hat{g}_{i}-P_{i}\right\|_{p}+2 N_{0 i} C_{p} \eta_{i} \leq \frac{1}{4} 2^{-n} \eta .
$$

Now putting

$$
\hat{f}_{i}=f \chi_{\Delta_{n}^{i}}-g_{i}+\hat{g}_{i}
$$

and

$$
\hat{f}=\sum_{i=1}^{2^{n}} \hat{f}_{i}
$$

we obtain similarly that for the function $\hat{f}$ and the polynomial $H=\sum_{i=1}^{2^{n}} H_{i}$ the conditions $a$ ) and $c$ ) of Lemma 2.9 hold.

Writing the analogue of inequality 43 for every $g_{i}$ and then summing over $i$, we prove condition $b$ ).

Also, for every $i, 1 \leq i \leq 2^{n}$, we can write the analogous condition of (44) as

$$
\sum_{k=N_{0 i}}^{N_{0 i+1}} a_{k i}^{2} \leq \frac{3}{\delta} \int_{\Delta_{n}^{i}}\left|g_{i}(x)\right|^{2} d x .
$$


Hence by the condition posed on the $L^{2}$-norms of the functions $g_{i}$ we conclude that

$$
\sup _{k, i}\left|a_{k i}\right|<\epsilon_{1} .
$$

Summing over $i$ both parts of the former inequality about the squares of the coefficients, we get

$$
\sum_{i=1}^{2^{n}} \sum_{k=N_{0 i}}^{N_{0 i+1}} a_{k i}^{2} \leq \frac{9}{\delta} \int_{0}^{1}|f(x)|^{2} d x .
$$

Using these estimates, we obtain

$$
\begin{gathered}
\sum_{k=N_{0 i}}^{N_{0 i+1}}\left|a_{k i}\right|^{2+\epsilon} \leq \sup _{k, i}\left|a_{k i}\right|^{\epsilon} \cdot \sum_{i=1}^{2^{n}} \sum_{k=N_{0 i}}^{N_{0 i+1}} a_{k i}^{2} \\
<\epsilon_{1}^{\epsilon} \cdot \frac{9}{\delta^{2}} \int_{0}^{1}|f(x)|^{2} d x<\epsilon .
\end{gathered}
$$

This gives condition $c^{*}$ ) in the lemma. Condition $d$ ) is completely analogous to that in Lemma 2.8 .

Proofs of Theorems 2.1 and 2.2. We will present here a detailed proof of Theorem 2.2, along with some indications of the changes needed to obtain Theorem 2.1.

Proof. Let $F$ be an everywhere dense sequence of functions in the space $C_{[0,1]}$, and let $R_{2}$ be the set of all rational numbers greater than or equal to 2 . In the case of Theorem 1.1, it suffices to take as $R_{2}$ a set consisting only of the point $\{p\}$. Denote by

$$
\Omega=F \times R_{2}=\left\{(f, r): f \in F \text { and } r \in R_{2}\right\}
$$

all possible pairs of elements of the sets $F$ and $R_{2}$. Let $\Omega=\left\{\omega_{j}\right\}_{j=1}^{\infty}$ be any enumeration of the set $\Omega$. For every $j \in \mathbf{N}$ take $\omega_{j}=\left(f_{j}, r_{j}\right)$, and denote

$$
f_{k}^{(j)}=f_{j} \quad \text { for } 1 \leq k \leq j .
$$

Then we apply Lemma 2.8 for $p=r_{j}$ and for every function $f_{k}^{(j)}, 1 \leq k \leq j$, with $\delta=2^{-k}, \eta=2^{-2 j}$ and $N_{0}=N_{j, k}$, where

$$
N_{1,1}=1, \quad N_{j+1,1}=M_{j, j}+1, \quad N_{j, k}=M_{j, k-1}+1 \quad \text { for } \quad 2 \leq k \leq j .
$$

Thus we get functions $\hat{f}_{k}^{(j)}$ and polynomials

$$
H_{k}^{(j)}=\sum_{i=N_{j, k}}^{M_{j, k}} a_{i} \phi_{i}
$$

such that

$$
\begin{gathered}
\left|\left\{x: \hat{f}_{k}^{(j)}(x)=f_{j}(x)\right\}\right| \geq 1-2^{-k}, \quad\left\|f_{j}-H_{k}^{(j)}\right\|_{r_{j}}<2^{-2 j}, \\
\left\|\hat{f}_{k}^{(j)}\right\|_{r_{j}}<2 \cdot 2^{\frac{k}{r_{j}^{\prime}}}\left\|f_{j}\right\|_{r_{j}} .
\end{gathered}
$$


Moreover, there exists a rearrangement $\pi_{j, k}$ of the numbers $N_{j, k}, N_{j, k}+1, \ldots, M_{j, k}$ such that

$$
\left\|\max _{N_{j, k} \leq m \leq M_{j, k}}\left|\sum_{i=N_{j, k}}^{m} a_{\pi_{j, k}(i)} \phi_{\pi_{j, k}(i)}(\cdot)\right|\right\|_{r_{j}} \leq C_{r_{j}} 2^{\frac{k}{r_{j}^{\prime}}}\left\|f_{j}\right\|_{r_{j}},
$$

where $C_{p}>0$ depends only on $p$.

Now, we define the desired rearrangement in our theorems. Let

$$
\sigma(n)=\pi_{j, k}(n) \quad \text { for } \quad N_{j, k} \leq n \leq M_{j, k} \text { and } 1 \leq k \leq j, j \in \mathbf{N} .
$$

It remains to prove that for the system $\Phi_{\sigma}=\left\{\phi_{\sigma(n)}\right\}_{n=1}^{\infty}$ the conclusions of Theorem 2.2 (and Theorem 1.1) are true; that is, if $2 \leq p<\infty, f \in L_{[0,1]}^{p}$ and $1>\epsilon>0$, we have to construct a function $f_{\epsilon}$ such that conditions (5) - (7) hold. Let $\nu \in \mathbf{N}$ be the least number for which

$$
2^{\nu}>\frac{1}{\epsilon}
$$

and let $\nu_{0} \in \mathbf{N}$ be any number such that

$$
2^{\nu_{0}}>\|f\|_{p}^{-1}, \quad \nu_{0} \geq \nu
$$

We now start the process of constructing the function $f_{\epsilon}$. As the first step we take an element $\omega_{j_{1}}=\left(f_{j_{1}}, r_{j_{1}}\right) \in \Omega$ such that

$$
\left\|f-f_{j_{1}}\right\|_{p}<2^{-2\left(\nu_{0}+1\right)}, \quad \text { where } j_{1}>\nu_{0}+1,
$$

and

$$
\left\|f_{j_{1}}\right\|_{r_{j_{1}}} \leq 2 \cdot\left\|f_{j_{1}}\right\|_{p}, \quad \text { where } r_{j_{1}} \geq p .
$$

((56), like (63) and (66) below, is obvious when $R_{2}=\{p\}$.) Then we denote

$$
g_{1}=\hat{f}_{\nu+1}^{\left(j_{1}\right)}
$$

and by (47)-(51) we conclude that

$$
\left|\left\{x: g_{1}(x)=f_{j_{1}}(x)\right\}\right| \geq 1-2^{-\nu-1}, \quad\left\|g_{1}-P_{1}\right\|_{r_{j_{1}}}<2^{-2 j_{1}},
$$

where

$$
P_{1}=\sum_{i=N_{j_{1}, \nu+1}}^{M_{j_{1}, \nu+1}} a_{\pi_{j_{1}, \nu+1}(i)} \phi_{\pi_{j_{1}, \nu+1}(i)}=H_{\nu+1}^{\left(j_{1}\right)}
$$

By $(56)$,

$$
\begin{gathered}
\left\|g_{1}\right\|_{p} \leq\left\|g_{1}\right\|_{r_{j_{1}}}<2 \cdot 2^{\frac{\nu+1}{p^{\prime}}}\left\|f_{j_{1}}\right\|_{r_{j_{1}}} \leq 4 \cdot 2^{\frac{\nu+1}{p^{\prime}}}\left\|f_{j_{1}}\right\|_{p}, \\
\left\|\max _{N_{j_{1}, \nu+1} \leq m \leq M_{j_{1}, \nu+1}}\left|\sum_{i=N_{j_{1}, \nu+1}}^{m} a_{\pi_{j_{1}, \nu+1}(i)} \phi_{\pi_{j_{1}, \nu+1}(i)}(\cdot)\right|\right\|_{p} \leq C_{p} 2^{\frac{\nu+1}{p^{\prime}}}\left\|f_{j_{1}}\right\|_{p} .
\end{gathered}
$$

We choose a second element $\omega_{j_{2}}=\left(f_{j_{2}}, r_{j_{2}}\right) \in \Omega$ so that

$$
\begin{gathered}
\left\|f-f_{j_{1}}-\left(P_{1}-g_{1}\right)-f_{j_{2}}\right\|_{p}<2^{-2\left(\nu_{0}+2\right)}, \quad \text { where } j_{2}>j_{1} ; \\
\left\|f_{j_{2}}\right\|_{r_{j_{2}}} \leq 2 \cdot\left\|f_{j_{2}}\right\|_{p}, \quad \text { where } r_{j_{2}} \geq p .
\end{gathered}
$$

Hence by (55) and (58) we have

$$
\left\|f_{j_{2}}\right\|_{p}<2^{-2 \nu_{0}}
$$


By induction, we take an element $\omega_{j_{n}}=\left(f_{j_{n}}, r_{j_{n}}\right) \in \Omega$ so that

$$
\begin{gathered}
\left\|f-\sum_{l=1}^{n-1} f_{j_{l}}-\sum_{l=1}^{n-1}\left(P_{l}-g_{l}\right)-f_{j_{n}}\right\|_{p}<2^{-2\left(\nu_{0}+n\right)}, \\
j_{n}>j_{n-1}>\cdots>j_{1}>\nu_{0}+1, \\
\left\|f_{j_{n}}\right\|_{r_{j_{n}}} \leq 2 \cdot\left\|f_{j_{n}}\right\|_{p}, \quad r_{j_{n}} \geq p,
\end{gathered}
$$

obtaining in that way

$$
\left\|f_{j_{n}}\right\|_{p}<2^{-2\left(\nu_{0}+n\right)}+2^{-2\left(\nu_{0}+n-1\right)}+2^{-2 j_{n-1}}<2^{-2\left(\nu_{0}+n-2\right)} .
$$

Hence, taking

$$
g_{n}=\hat{f}_{\nu+n}^{\left(j_{n}\right)}
$$

by (47)-(51) and (66) we get

$$
\left|\left\{x: g_{n}(x)=f_{j_{n}}(x)\right\}\right|>1-2^{-\nu-n}, \quad\left\|g_{n}-P_{n}\right\|_{p}<2^{-2 j_{n}},
$$

where

$$
P_{n}=\sum_{i=N_{j_{n}, \nu+n}}^{M_{j_{n}, \nu+n}} a_{\pi_{j_{n}, \nu+n}}(i) \Phi_{j_{n}, \nu+n}(i)=H_{\nu+n}^{\left(j_{n}\right)}
$$

Also

$$
\begin{gathered}
\left\|g_{n}\right\|_{p}<4 \cdot 2^{\frac{\nu+n}{p^{\prime}}}\left\|f_{j_{n}}\right\|_{p}, \\
\left\|\max _{N_{j_{n}, \nu+n} \leq m \leq M_{j_{n}, \nu+n}}\left|\sum_{i=N_{j_{n}, \nu+n}}^{m} a_{\pi_{j_{n}}(i)} \phi_{\pi_{j_{n}}(i)}(\cdot)\right|\right\|_{p} \leq C_{p} 2^{\frac{\nu+n}{p^{\prime}}}\left\|f_{j_{n}}\right\|_{p} .
\end{gathered}
$$

By (67), (68) and (70) we see immediately that the series

$$
\sum_{n=1}^{\infty} f_{j_{n}}, \quad \sum_{n=1}^{\infty} g_{n}, \quad \sum_{n=1}^{\infty} P_{n}
$$

converge in the $L^{p}$-metric and

$$
\left|\left\{x: \sum_{n=1}^{\infty} g_{n}(x)=\sum_{n=1}^{\infty} f_{j_{n}}(x)\right\}\right|>1-2^{-\nu} .
$$

From (65) we deduce that

$$
f=\sum_{n=1}^{\infty} f_{j_{n}}-\sum_{n=1}^{\infty} g_{n}+\sum_{n=1}^{\infty} P_{n}
$$

Hence we easily conclude from (72) and (53) that for the function

$$
f_{\epsilon}=\sum_{n=1}^{\infty} P_{n}
$$

condition (5) holds. Also, we can check by (67) and (71) that the series in (6) converges. In order to prove (7) we select for a given natural number $k$ the corresponding numbers $N_{j_{\ell}, \nu+\ell}$ and $M_{j_{\ell}, \nu+\ell}$ such that

$$
N_{j_{\ell}, \nu+\ell} \leq k \leq M_{j_{\ell}, \nu+\ell} .
$$


Then, using the triangle inequality, by (67), (71) and (58) we obtain

$$
\begin{gathered}
\left\|S_{\Phi, k}^{*}\left(f_{\epsilon}, \cdot\right)\right\|_{p} \leq \sum_{n=1}^{\ell}\left\|\max _{N_{j_{n}, \nu+n} \leq m \leq M_{j_{n}, \nu+n}}\left|\sum_{i=N_{j_{n}, \nu+n}}^{m} a_{\pi_{j_{n}}(i)} \phi_{\pi_{j_{n}}(i)}(\cdot)\right|\right\|_{p} \\
\leq C_{p} \sum_{n=1}^{\ell} 2^{\frac{\nu+n}{p^{\prime}}}\left\|f_{j_{n}}\right\|_{p} \leq C_{p}\left(\left\|g_{1}\right\|_{p}+2^{-2 j_{1}}+\sum_{n=2}^{\ell} 2^{\frac{\nu+n}{p^{\prime}}} 2^{-2\left(\nu_{0}+n\right)}\right) .
\end{gathered}
$$

We can estimate the last expression by (53)-(58) and the condition $b$ ) of Lemma 2.8. That finishes the proof of (7) and, therefore, of the first part of Theorem 2.2. (In particular, we have completed the proof of Theorem 2.1 too.)

It remains to show then how one can get the uniform estimates in the second part of the conclusion of Theorem 2.2- that is, if $f \in L_{[0,1]}^{p}$ for all $p>2$ and $\epsilon>0$ is given, then there exists a function $f_{\epsilon} \in \bigcap_{2 \leq r<\infty} L_{[0,1]}^{r}$ such that conditions (5) - (7) for the system $\Phi_{\sigma}=\left\{\phi_{\sigma(n)}\right\}_{n=1}^{\infty}$ are true for all $p>2$. To do this, we fix as above the least number $\nu$ for which (53) holds. Then we take any sequence of increasing rational numbers tending to infinity:

$$
p_{1}<p_{2}<\cdots<p_{n}<\cdots ; \quad p_{n} \rightarrow \infty .
$$

The idea is to apply now the previous arguments to each $p=p_{n}$, inductively in $n$, but as if we were in the situation of Theorem 1.1. As the first step we take an element $\omega_{j_{1}}=\left(f_{j_{1}}, r_{j_{1}}\right) \in \Omega$, with $r_{j_{1}}=p_{1}$, such that condition (55) holds. (Condition (56) is superfluous here.) Then, as before, we take $g_{1}=\hat{f}_{\nu+1}^{\left(j_{1}\right)}$ for which the conditions (58)-(61) hold with $p_{1}$ instead of $p$. We choose the second element $\omega_{j_{2}}=\left(f_{j_{2}}, r_{j_{2}}\right) \in \Omega$, with $r_{j_{2}}=p_{2}$, so that condition (62) holds with $p_{2}$ instead of $p$. Hence by (55) and (58) we get

$$
\left\|f_{j_{2}}\right\|_{p_{1}}<2^{-2 \nu}
$$

At the $n$-th step we take an element $\omega_{j_{n}}=\left(f_{j_{n}}, r_{j_{n}}\right) \in \Omega$, with $r_{j_{n}}=p_{n}$, so that

$$
\left\|f-! \sum_{l=1}^{n-1} f_{j_{l}}-\sum_{l=1}^{n-1}\left(P_{l}-g_{l}\right)-f_{j_{n}}\right\|_{p_{n}}<2^{-2(\nu+n)}, \quad j_{n}>j_{n-1}>\cdots>j_{1}>\nu+1,
$$

obtaining immediately that

$$
\left\|f_{j_{n}}\right\|_{p_{n-1}}<2^{-2\left(\nu_{0}+n-2\right)}
$$

Now, taking $g_{n}=\hat{f}_{\nu+n}^{\left(j_{n}\right)}$ as above, we see that conditions (68)-(71) hold with $p_{n}$ instead of $p$. The rest of the proof is easy. Given any $p>2$, by (73) we can select $n \in \mathbf{N}$ such that $p_{n-1}>p$, and use the same arguments, in this case referring to condition (74) instead of (67).

Analyzing the proofs of Theorems 2.1 and 2.2 above, the reader can easily see that what we have proved (say in the context of Theorem 2.2) is the following fact:

For every $\epsilon>0,2<p<\infty$, and every $f \in L_{[0,1]}^{p}$ we have constructed a function $f_{\epsilon} \in L_{[0,1]}^{p}$ such that it differs from $f$ only on a set that has measure less than $\epsilon$ and whose Fourier series with respect to the system $\Phi_{\sigma}$ converges almost everywhere and in the metric of $L_{[0,1]}^{p}$. Indeed, it is a subseries of a fixed series (see (47)-(52)), 
namely,

$$
\sum_{n=1}^{\infty} a_{\sigma(n)} \phi_{\sigma(n)} .
$$

So if we repeat the proofs of Theorems 2.1 and, respectively, 2.2 using Lemma 2.9 instead of Lemma 2.8, and we adequately estimate the coefficients in every step, then we obtain Theorem 2.3 and, respectively, Theorem 2.4.

\section{REFERENCES}

[B] Bari, N.K., A treatise on trigonometric series, Macmillan, New York, 1964. MR 30:1347

[GKK] Garcia-Cuerva, J., Kazarian, K.S. and Kazarian, S.S., On Men'shov's C-strong property, Acta Sci. Math. (Szeged) 58 (1993), 249-256. MR 95g:42048

[G] Garsia, A. M., Topics in almost everywhere convergence, Markham, Chicago, 1970. MR 41:5869

[GW] Goffman C. and Waterman, D., Some aspects of Fourier series, Amer.Math. Monthly 77 (1970), 119-133. MR 40:6155

[Gr] Grigorian, M.G., About some properties of orthogonal systems, Izv. Ross. Akad. Nauk Ser. Mat. 57 (1993), no. 5, 75-105; English transl., Russian Acad. Sci. Izv. Math. 43 (1994), 261-290. MR 95c:42028

[K1] Katznelson, Y., On a theorem of Menchoff, Proc. Amer. Math. Soc. 53 (1975), 396-398. MR 52:1194

[K2] Katznelson, Y., An introduction to harmonic analysis, Wiley, New York, 1968. MR 40:1734

[Ka] Kazarian, K.S., On some questions of the theory of orthogonal series, Mat. Sb. 119 (1982), 278-294; English transl., Math. USSR-Sb. 47 (1984), 269-285. MR 84m:42035

[KS] Kazarian, K.S. and Soria, F., On Fourier series of orthogonal systems for modified functions, C.R. Acad. Sci. Paris Ser. I. Math. 320 (1995), 9-14. MR 96b:42030

[Ko] Kozlov, V. Ya., On complete systems of orthogonal functions, Mat. Sb. 26(68) (1950), 351-364. (Russian) MR 12:174g

[L] Luzin, N. N., Integral and trigonometric series, 1916, Dissertation, Moscow; augmented reprint, GITTL, Moscow, 1951. (Russian). MR 14:2g

[M1] Menchoff, D. [Men'shov D.E.], Sur l'unicité du développement trigonométrique, C.R. Acad. Sci. Paris 163 (1916), 433-436.

[M2] Menchoff, D. [Men'shov D.E.], Sur les séries de Fourier des fonctions continues, Mat. Sb. 8(50) (1940), 493-518. MR 2:189g

[M3] Menchoff, D. [Men'shov D.E.], Sur la représentation des fonctions mesurables par des séries trigonométriques, Mat. Sb. 9(51) (1941), 667-692. MR 3:106a

[M4] Menchoff, D. [Men'shov D.E.], Sur les sommes partielles des séries trigonométriques, Mat. Sb. 20(62) (1947), 197-236. (Russian; French summary) MR 8:577a

[O] Olevskii, A., the existence of functions with unremovable Carleman singularities, Dokl. Akad. Nauk SSSR 238 (1978), 796-799, English transl. in Soviet Math. Dokl. 19(1978). MR 57:7005

[Or] Orlicz, W., Über die unabhängig von der Anordnung fast überall konvergenten Reihen, Bull. Acad. Polon. Sci. 81 (1927), 117-125.

[P] Pogosian, N.B., Representation of measurable functions by orthogonal series, Mat. Sb. 98(140) (1975), 102-112; English transl., Math. USSR-Sb. 27 (1975), 93-102. MR 58:6901a

[T] Talalian, A.A., Representation of measurable functions by series, Uspekhi Mat. Nauk 15 5(95) (1960), 77-141, English transl. in Russian Math. Surveys 15 (1960). MR 23:A2704

[U] Ul'yanov, P.L., Luzin's work on the metric theory of functions, Russian Math. Surveys 40:3 (1985), 15-77. MR 86k:01040

[U1] Ul'yanov, P.L., Solved and unsolved problems in the theory of trigonometric and orthogonal series, Uspekhi Mat. Nauk 19 1(115) (1964), 3-69, English transl. in Russian Math. Surveys 19 (1964). MR 28:4294 
3798 MARTIN G. GRIGORIAN, KAZAROS S. KAZARIAN, AND FERNANDO SORIA

[U2] Ul'yanov, P.L., On unconditional convergence and summation, Izvestiya AN SSSR Ser. Math. 22 (1958), 811-840. (Russian) MR 21:2151

[Z] Zygmund, A., Trigonometric series, vol. 1, Cambridge Univ. Press, 1959. MR 21:6498

Department of Radiophysics, Yerevan State University, Yerevan 375049, Armenia

E-mail address: gmartin@ysu.am

Departamento de Matemáticas, Facultad de Ciencias, Universidad Autónoma de MADRID, 28049 MADRID, SPAIN

E-mail address: kazaros.kazarian@uam.es

Departamento de Matemáticas, Facultad de Ciencias, Universidad Autónoma de MADRID, 28049 MADRID, SPAIN

E-mail address: fernando.soria@uam.es 